\title{
Direct Switching Position Control Algorithms for Pneumatic Actuators using On/Off Solenoid Valves
}

\author{
Yile Zhang and Gary M. Bone* \\ Department of Mechanical Engineering \\ McMaster University \\ Hamilton, Canada \\ *Corresponding author. E-mail: gary@ mcmaster.ca
}

\begin{abstract}
Pneumatic actuators are advantageous in terms of cost, power to weight ratio and inherent safety. However, their dynamics makes precise closed-loop position control very difficult in practice. Two sliding-mode control algorithms for controlling the position of a pneumatic cylinder by directly switching four on/off solenoid valves are proposed in this paper. The solenoid valves are much less expensive than the commonly used servo or proportional valves. The proposed algorithms are compared to two state of the art position control algorithms. Based on experiments on a high friction cylinder with various payloads, the proposed controllers provide superior performance in terms of valve switches per second, steady state error, settling time and overshoot. The achieved number of valve switches per second is also about one tenth of the number required by the pulse-width modulation method that is commonly used with on/off valves. This should result in prolonged valve lifetimes and reduced maintenance costs.
\end{abstract}

Keywords-actuator; pneumatic; position control; sliding-mode control; solenoid valve.

\section{INTRODUCTION}

Pneumatic actuators are widely used in automation because they provide several advantages. They are low cost, clean and provide a high power to weight ratio. They are also inherently safe due to their natural compliance. However, the closed-loop control of their position for servoing applications is very difficult and remains an active research topic (e.g. [1]-[5]). Our interest is on the closed-loop position of pneumatic cylinders. The majority of previous papers employed proportional or servo valves to control the flow of air to/from the cylinder's two chambers. In this paper, on/off solenoid valves will be used since they are much less expensive than proportional/servo valves (e.g., US $\$ 40$ vs. US\$800). This advantage is offset by the disadvantage that their flow behaviour is discontinuous, making smooth and precise position control more difficult to achieve. While pulse-width modulation (PWM) may be used with on/off valves to approximate a proportional valve in position control applications (e.g.,[6]-[8]) it also causes frequent valve switching leading to shorter valve life. In

Research sponsored by the Natural Sciences and Engineering Research Council of Canada (NSERC) through a Discovery Grant. contrast, systems employing the direct switching method only switch the valves when it is necessary for the desired closedloop performance. This reduced switching frequency prolongs the valve life, leading to reduced repair and replacement costs. In spite of its advantages, relatively few researchers have investigated the direct switching approach.

In [9], a double acting cylinder was controlled using four two-way on/off solenoid valves (i.e. two valves per cylinder chamber) and an optical encoder for position feedback. Their sliding-mode control (SMC) algorithm used three operating modes for the valves, and was the first to consider the problem of excessive valve switching. This algorithm will be further described in section III. For a $2 \mathrm{~kg}$ payload and a $40 \mathrm{~mm}$ step input, the steady state error (SSE) was $0.1 \mathrm{~mm}$ and the overshoot (OS) was less than $10 \mathrm{~mm}$. They did not report any robustness results. A more sophisticated SMC algorithm with seven valve operating modes was proposed in [10]. They used the same valve configuration as in [9]. The introduction of the four additional modes and two additional tuning parameters was shown to improve both the tracking errors and valve switching frequency. For a $0.9 \mathrm{~kg}$ payload and $40 \mathrm{~mm}$ step input the 7-mode controller reduced the positive OS from 7.2 $\mathrm{mm}$ to $0.76 \mathrm{~mm}$ (i.e., $89 \%$ reduction) compared with the 3mode controller from [9]. For the same payload and a multiple sine wave reference input the switching frequency with the 7mode controller was $48 \%$ less than with the 3-mode controller, while the tracking errors were similar. Nonlinear model predictive control algorithms were used to directly switch the valves in [11] and [12]. While these algorithms have the potential to outperform those from [9] and [10] they require a system model and greater computing power to implement.

In this paper we propose modified versions of the SMC algorithms from [9] and [10], and compare their experimental position control performance with the original algorithms. The system structure is described in section II. Next, the designs of the existing and proposed control algorithms are presented. Experimental results, including robustness tests, are presented in section IV. Finally, conclusions are drawn in section V. 


\section{SYSTEM STRUCTURE}

A schematic of the system structure is shown in Fig. 1. As in [9] and [10], two two-way on/off valves (also termed 2/2 valves) are connected to each chamber of the cylinder. This has the advantage of allowing the pressure of each chamber to be independently controlled. The cylinder drives a payload mass horizontally. The position and cylinder pressures are fed back to PC-based control system. The PC switches the valves via optocouplers.

\section{CONTROLLER DESIGN}

\section{A. Three-mode Sliding Mode Control Algorithm}

In this section, the three-mode sliding mode controller (abbreviated as SMC3) proposed by Nguyen et al. [9] is briefly summarized. The three operating modes are defined as follows:

Mode 1: Both chambers' valves are closed

$\left(u_{1}=0, u_{2}=0, u_{3}=0\right.$ and $\left.u_{4}=0\right)$

Mode 2: Chamber A charges and chamber B discharges

$\left(u_{1}=1, u_{2}=0, u_{3}=0\right.$ and $\left.u_{4}=1\right)$

Mode 3: Chamber A discharges and chamber B charges $\left(u_{1}=0, u_{2}=1, u_{3}=1\right.$ and $\left.u_{4}=0\right)$

Mode 2 is used to move the piston in the positive direction and mode 3 is used to move it in the negative direction. Mode 1 is used to reduce the chattering when the tracking error is small enough and to save energy.

They use the second-order sliding surface:

$$
s=\frac{\ddot{e}}{\omega^{2}}+\frac{2 \zeta \dot{e}}{\omega}+e
$$

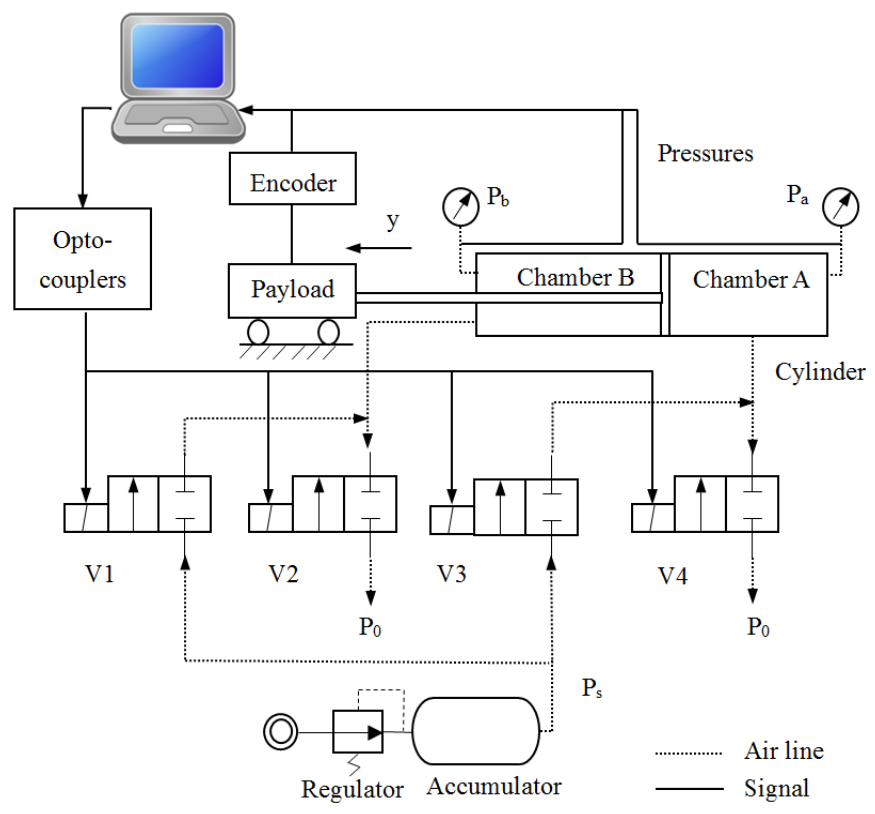

Figure 1. System schematic diagram. where $e=y-y_{d}$ is the position error, $y$ is the actual position, $y_{d}$ is the desired position, and $\zeta$ and $\omega$ are constant and positive numbers. The value of $s$ determines the mode according to:

$$
\begin{cases}s>\varepsilon, & \text { mode } 3 \\ s<-\varepsilon, & \text { mode } 2 \\ -\varepsilon \leq s \leq \varepsilon, & \text { mode } 1\end{cases}
$$

where $\mathcal{E}$ is the mode 1 boundary limit. It is used to reduce the valve switching caused by control chattering. To limit the tracking error it is desirable to choose $\varepsilon$ as small as possible. However, $\varepsilon$ also needs to be large enough to reduce chattering.

\section{B. Seven-mode Sliding Mode Control Algorithm}

The seven-mode sliding mode controller (abbreviated as SMC7) proposed by Hodgson et al. [10] is briefly summarized in this section. They extended SMC3 from three to seven operating modes with the goal of reducing the number of valve switches per second. The valve states for the seven modes are defined in Table 1. Modes 1, 6 and 7 are the same as SMC3 modes 1, 2 and 3, respectively. Modes 2-5 are new.

As with SMC3 the sliding surface is defined by (1). They extended the three regions of $s$ used with SMC3 (see (2)) to five regions. The regions, corresponding modes and qualitative forces are listed in Table 2 . In addition to deadband $\mathcal{E}$, they introduce a larger boundary limit $\beta$. Examining Table 2, regions $s \leq-\beta,-\varepsilon<s \leq \varepsilon$ and $s>\beta$ map to unique modes. Regions $-\beta<s \leq-\varepsilon$ and $\varepsilon<s \leq \beta$ map to two modes each, and additional logic based on the chamber pressures are required to identify the unique mode to apply. The principle behind the mode selection is the mode producing the larger acceleration should be chosen since it will reduce the tracking error faster.

For region $-\beta<s \leq-\varepsilon$, they deduced that the steady state payload acceleration with mode 2 is roughly proportional to $P_{s}-P_{b}$ where $P_{s}$ is the supply pressure and $P_{b}$ is the pressure inside chamber B. Similarly, with mode 4 the steady state acceleration is roughly proportional to $P_{a}-P_{0}$ where $P_{a}$ is the pressure inside chamber $\mathrm{A}$ and $P_{0}$ is atmospheric pressure. They then defined

$$
E_{1}=\left(P_{s}-P_{b}\right)-\left(P_{a}-P_{0}\right)
$$

where if $E_{1}$ is positive mode 2 should produce a larger acceleration than mode 4 . So the following switching logic is

TABLE I. VALVE STATES FOR THE SEVEN OPERATING MODES USED BY THE SMC7 CONTROLLER

\begin{tabular}{|c|c|c|c|c|c|c|c|}
\hline \multirow{2}{*}{ Valve } & \multicolumn{7}{|c|}{ Mode } \\
\cline { 2 - 8 } & $\mathbf{1}$ & $\mathbf{2}$ & $\mathbf{3}$ & $\mathbf{4}$ & $\mathbf{5}$ & $\boldsymbol{6}$ & $\mathbf{7}$ \\
\hline$u_{1}$ & 0 & 1 & 0 & 0 & 0 & 1 & 0 \\
\hline$u_{2}$ & 0 & 0 & 1 & 0 & 0 & 0 & 1 \\
\hline$u_{3}$ & 0 & 0 & 0 & 0 & 1 & 0 & 1 \\
\hline$u_{4}$ & 0 & 0 & 0 & 1 & 0 & 1 & 0 \\
\hline
\end{tabular}


applied in the region $-\beta<s \leq-\varepsilon$ :

$$
\begin{cases}E_{1}>0, & \text { mode } 2 \\ E_{1} \leq 0, & \text { mode } 4\end{cases}
$$

For region $\varepsilon<s \leq \beta$, a similar approach is employed. Note that the steady state acceleration will be negative. They defined

$$
E_{2}=\left(P_{s}-P_{a}\right)-\left(P_{b}-P_{0}\right)
$$

where if $E_{2}$ is positive mode 5 should produce a larger acceleration than mode 3 . So the following switching logic is applied in the region $-\beta<s \leq-\varepsilon$ :

$$
\begin{cases}E_{2}>0, & \text { mode } 5 \\ E_{2} \leq 0, & \text { mode } 3\end{cases}
$$

In order to reduce the number of switches between modes when (5) and (6) are applied, they introduced a timeout parameter $\tau$. Mode 2, 3, 4 or 5 is maintained for a period of $\tau$ or longer. A larger $\tau$ therefore reduces the switching but may also lead to larger tracking errors.

\section{Proposed Sliding Mode Control Algorithms}

We propose two SMC algorithms in this section. Both are modifications of the existing algorithms, and are based on the idea that adding integral action can reduce settling time and SSE if it is properly applied.

In order to add integral action, the sliding surface is based on $\int_{0}^{t} e d t$ rather than $e$. Thus, we define the new sliding surface as:

$$
s=\frac{\ddot{e}}{\omega^{3}}+\frac{3 \zeta \dot{e}}{\omega^{2}}+\frac{3 \zeta^{2} e}{\omega}+\int_{0}^{t} e d t
$$

where $\int_{0}^{t} e d t$ provides the integral action. Integral windup may occur when there is a large change in the desired position or a large disturbance, and the integral term accumulates a significant error during the transient. The time required to reduce this large integral term can lead to overshoot and a longer settling time. Anti-windup is implemented by bounding the integral action as follows:

$$
\left|\int_{0}^{t} e d t\right| \leq e_{\text {limit }}
$$

The first proposed algorithm, termed integral SMC3

TABLE II. FIVE REGIONS OF $S$ USED BY THE SMC7 CONTROLLER

\begin{tabular}{|c|c|c|}
\hline Region & Corresponding Modes & $\begin{array}{c}\text { Qualitative Pneumatic } \\
\text { Force }\end{array}$ \\
\hline$s \leq-\beta$ & 6 & Large positive \\
\hline$-\beta<s \leq-\varepsilon$ & 2 or 4 & Small positive \\
\hline$-\varepsilon<s \leq \varepsilon$ & 1 & Zero \\
\hline$\varepsilon<s \leq \beta$ & 3 or 5 & Small negative \\
\hline$s>\beta$ & 7 & Large negative \\
\hline
\end{tabular}

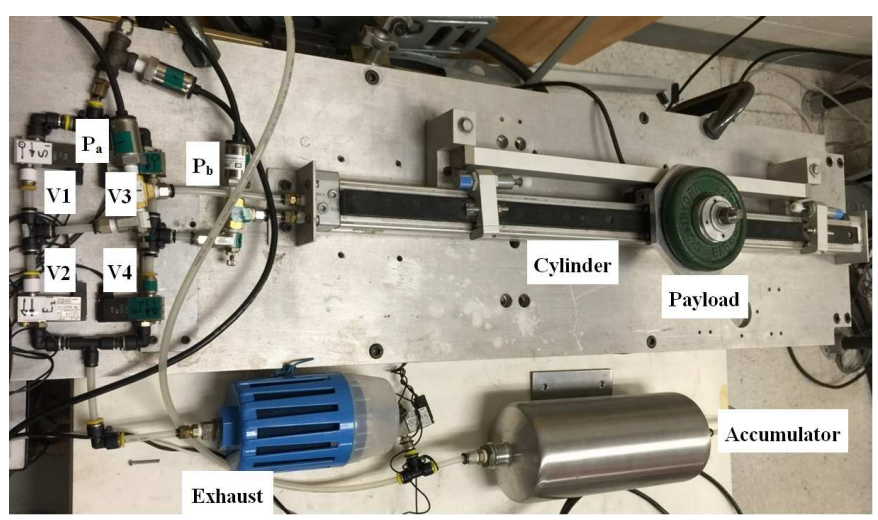

Figure 2. System hardware (PC and optocouplers are not shown).

(ISMC3), is derived from SMC3 by replacing (1) with (7) and (8). The second proposed algorithm, termed integral SMC7 (ISMC7), is similarly derived from SMC7 by replacing (1) with (7) and (8).

\section{EXPERIMENTS}

\section{A. Hardware}

The hardware implementation of the system structure presented in section II is shown in Fig. 2. The cylinder (Festo, model number DGPL-25-600) is rodless; and has a $600 \mathrm{~mm}$ stroke and $25 \mathrm{~mm}$ bore. The four on/off valves are made by MAC, model number 34B-AAA-GDFB-1BA. The linear encoder has a resolution of $0.01 \mathrm{~mm}$. The pressure sensors are SSI Technologies, model number P51-100-A-B-I36-5V-000000 . The supply pressure is regulated at $0.6 \mathrm{MPa}$. All of the sensor signals and control signals are interfaced with the PC using a National Instruments PCIe-6365 card. The PC runs 64bit Windows 7 with a $3.10 \mathrm{GHz}$ Intel i5-2400 processor and 8.00 GB RAM. This data acquisition and control system is programmed in $\mathrm{C}$ and operates at a $1 \mathrm{kHz}$ sampling frequency.

\section{B. Experimental Results and Discussion}

To test both the transient and steady state performance, the desired position trajectory consists of a series of step changes. The step heights are $200 \mathrm{~mm},-200 \mathrm{~mm}, 100 \mathrm{~mm}$ and $10 \mathrm{~mm}$. The total duration is $4 \mathrm{~s}$. The controller parameters were tuned for a nominal payload of $2.14 \mathrm{~kg}$.

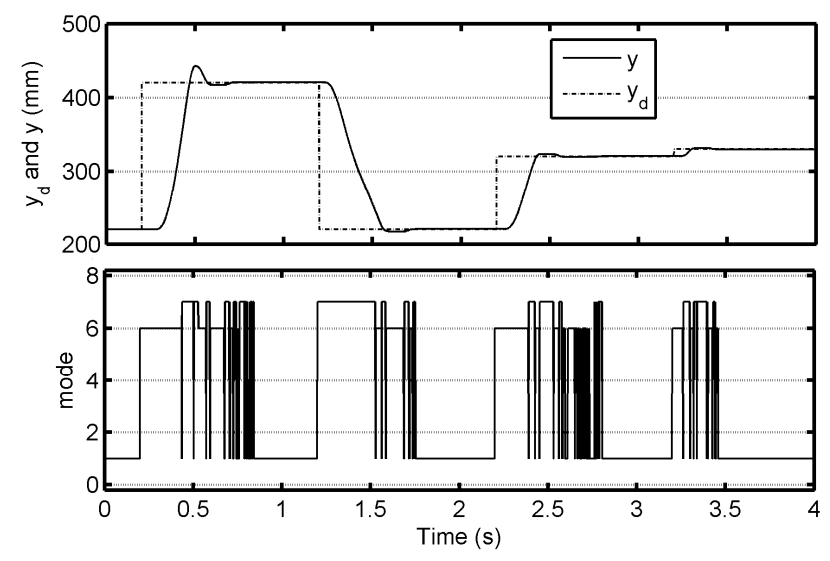

Figure 3. Experimental result for SMC3 with the nominal payload. 

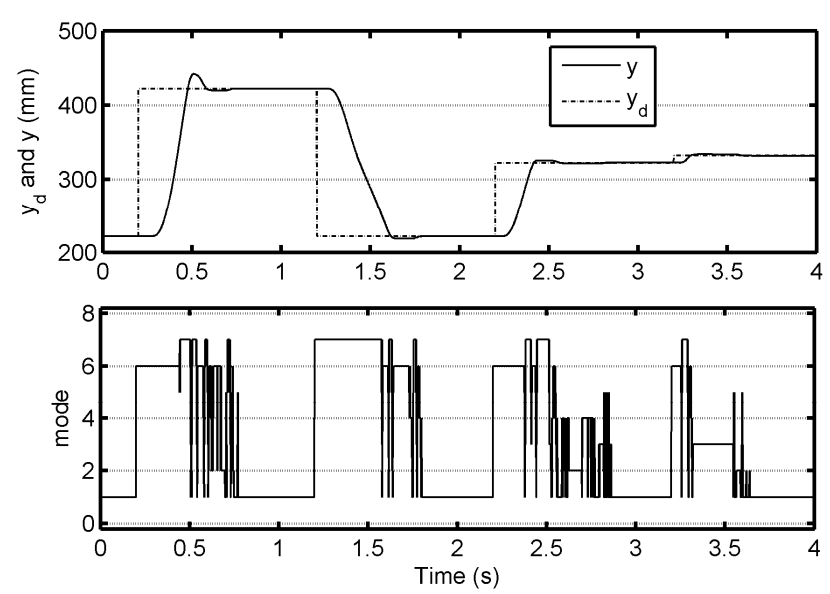

Figure 4. Experimental result for SMC7 with the nominal payload.

Sample responses for the four controllers with the nominal payload are plotted in Figs. 3-6. In these figures SMC3 and ISMC3 modes 2 and 3 are plotted as 6 and 7 (which are equivalent in terms of the valve states) to allow the 3 -mode and 7-mode plots to be more easily compared.

Comparing Figs. 3 and 4 the responses obtained with SMC3 and SMC7 appear to be quite similar in terms of their position tracking and number of mode switches. In contrast, the performance of ISMC3 (Fig. 5) is clearly better than SMC3 (Fig. 3). ISMC3 exhibits lower OS and longer periods were no mode switches occur. ISMC7 (Fig. 6) demonstrates similar improvements over SMC7 (Fig. 4). As with SMC3 and SMC7, the benefits of ISMC7 relative to ISMC3 are not obvious from these plots. A quantitative comparison based on numerous experiments will be discussed next.

In addition to tests with the nominal payload, tests were performed with a decreased payload $(0.95 \mathrm{~kg})$ and an increased payload $(3.24 \mathrm{~kg})$ to quantify each controller's robustness to payload mismatch. The performance metrics include the SSE, OS, and the settling time $t_{s}$, which is defined here as the time for the response to settle within $1 \mathrm{~mm}$ of the desired steady state position. These were averaged over the four step changes. The final metric is the average number of times each valve is switched per second (SPS). Five tests were

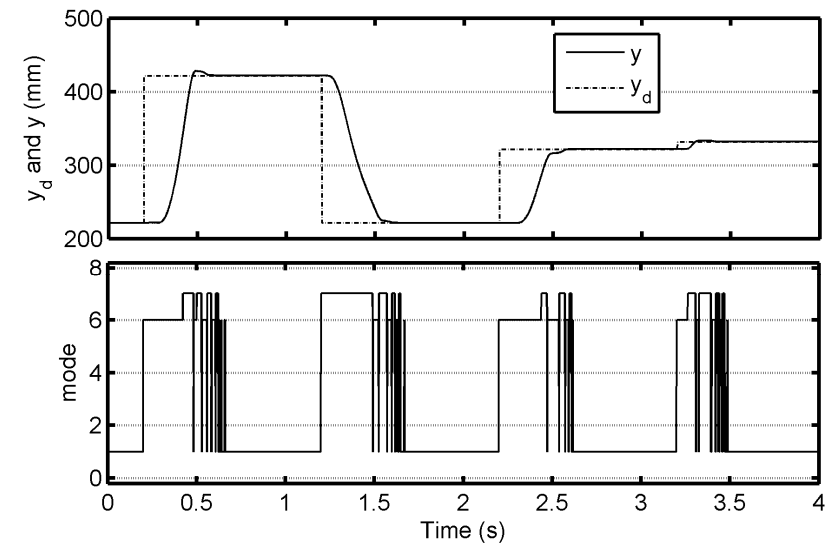

Figure 5. Experimental result for ISMC3 with the nominal payload.
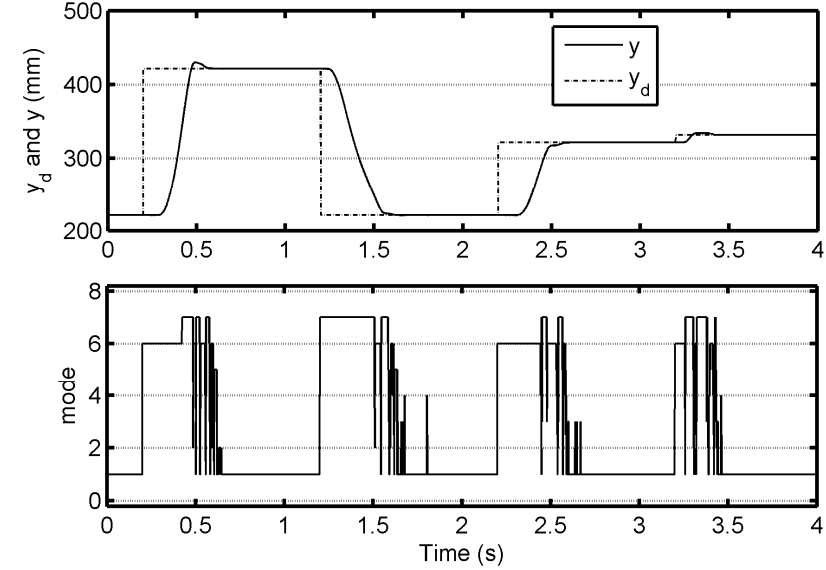

Figure 6. Experimental result for ISMC7 with the nominal payload. performed with each controller and payload combination. The averaged results of these tests are reported in Table III.

Examining the Table III results for the nominal payload, the SPS of SMC7 is $18 \%$ smaller than that of SMC3. For SSE, $t_{s}$ and OS the differences are $-6 \%,+10 \%$ and $-22 \%$, respectively. While SMC7 demonstrates improved performance vs. SMC3, the improvement is modest compared to the results reported in [10]. It is hypothesized that the much larger static friction due to the seals of the Festo DGPL cylinder used in our experiments $(\sim 80 \mathrm{~N}$ vs. $\sim 0.3 \mathrm{~N}$ for the Airpel M16 cylinder used in [10]) is the reason for the modest improvements observed here for SMC7.

The tabulated results for both ISMC3 and ISMC7 are consistently superior to the SMC3 and SMC7 results. For example, with the nominal payload the reductions in SPS, SSE, $t_{s}$ and OS for ISMC3 vs. SMC3 were 37\%, 57\%, 15\% and $65 \%$, respectively. The improvements with the altered payloads were smaller. For example, with the increased payload, ISMC3 reduced SPS, SSE, $t_{s}$ and OS by $43 \%, 19 \%$, $16 \%$ and $47 \%$ respectively, compared with SMC3. The improvements in SSE and $t_{s}$ were expected due to the integral

TABLE III. AVERAGED EXPERIMENTAL RESULTS

\begin{tabular}{|c|c|c|c|c|c|}
\hline Controller & Payload & SPS & $\begin{array}{c}\text { SSE } \\
(\mathbf{m m})\end{array}$ & $\begin{array}{c}\mathbf{t}_{\mathbf{s}} \\
(\mathbf{s})\end{array}$ & $\begin{array}{c}\text { OS } \\
(\mathbf{m m})\end{array}$ \\
\hline SMC3 & Nominal & 17.85 & 0.35 & 0.40 & 7.99 \\
\hline SMC3 & Decreased & 19.50 & 0.21 & 0.34 & 2.87 \\
\hline SMC3 & Increased & 20.35 & 0.26 & 0.45 & 12.53 \\
\hline SMC7 & Nominal & 14.60 & 0.33 & 0.44 & 6.20 \\
\hline SMC7 & Decreased & 16.03 & 0.28 & 0.42 & 2.43 \\
\hline SMC7 & Increased & 18.60 & 0.40 & 0.47 & 12.77 \\
\hline SMC13 & Nominal & 11.20 & 0.15 & 0.34 & 2.78 \\
\hline SMC13 & Decreased & 14.05 & 0.17 & 0.35 & 0.78 \\
\hline SMC13 & Increased & 11.65 & 0.21 & 0.38 & 6.61 \\
\hline SMCI7 & Nominal & 9.65 & 0.19 & 0.34 & 3.28 \\
\hline SMCI7 & Decreased & 11.88 & 0.20 & 0.35 & 1.14 \\
\hline SMCI7 & Increased & 8.55 & 0.17 & 0.38 & 6.60 \\
\hline
\end{tabular}


action included in ISMC3, but the improvements in the other two metrics were not. The results also show that the performances of ISMC7 and ISMC3 are similar, except that ISMC7 lowered the SPS. For example, with the increased payload the SPS was $27 \%$ smaller with ISMC7. Lastly, the results with the altered payloads demonstrate the SPS, SSE and $t_{s}$ metrics were robust to changing the payload, but the OS was not.

\section{CONCLUSION}

We have proposed two control algorithms, ISMC3 and ISMC7, for controlling the position of a pneumatic cylinder by directly switching the on/off solenoid valves. The proposed algorithms experimentally outperform the existing algorithms in terms of the SPS, SSE, $t_{s}$ and OS performance metrics. The proposed algorithms also switch the valves much less than if PWM was used. For example, the PWM period used in [7] produces an SPS of 100 (assuming the duty cycle is not equal to 0 or $100 \%$ ). This is 10 times larger than the SPS achieved by ISMC7 in our experiments. This reduced switching should prolong the life of the valves, and lower maintenance costs. Since the performances of ISMC3 and ISMC7 are similar, it is recommended that ISMC3 be used since it does not require any pressure sensing.

\section{REFERENCES}

[1] R. A. Rahman, L. He and N. Sepehri, "Design and experimental study of a dynamical adaptive backstepping-sliding mode control scheme for position tracking and regulating of a low-cost pneumatic cylinder," Int. J. Robust Nonlin., vol. 26, pp. 853-875, 2016.

[2] V. Nazari and B. Surgenor, "Improved position tracking performance of a pneumatic actuator using a fuzzy logic controller with velocity, system lag and friction compensation," Int. J. Control Autom., vol. 14, pp. 1376-1388, 2016.

[3] R. A. Rahman and N. Sepehri, "Pneusimulator: A simulation software for studying performance of controllers in pneumatic actuating systems," Int. J. Fluid Power, vol. 18, pp. 70-72, 2017.

[4] L. Zhao, Y. Xia, Y. Yang and Z. Liu, "Multicontroller Positioning Strategy for a Pneumatic Servo System Via Pressure Feedback," IEEE Trans. Ind. Electron., vol. 64, pp. 4800-4809, 2017.

[5] F. Soleymani, S. M. Rezaei, M. Zareinejad, K. Baghestan and A. Rahimi, "Position control of a servo-pneumatic actuator with mismatched uncertainty using multiple-surface sliding mode controller and high-gain observer," Trans. Inst. Meas. Control, vol. 39, pp. 1497-1508, 2017.

[6] R. B. van Varseveld and G. M. Bone, "Accurate position control of a pneumatic actuator using on/off solenoid valves", IEEE/ASME Trans. Mechatron., vol. 2, no. 3, pp. 195-204, 1997.

[7] K. Ahn and S. Yokota, "Intelligent switching control of pneumatic actuator using on/off solenoid valves," Mechatronics, vol. 15, pp. 683702, 2005.

[8] X. Shen, J. Zhang, E. Barth, and M. Goldfarb, "Nonlinear model-based control of pulse width modulated pneumatic servo systems", ASME J. Dyn. Syst. Meas. Control, vol. 128, pp. 663-669, 2006

[9] T. Nguyen, J. Leavitt, F. Jabbari, and J. E. Bobrow, "Accurate sliding mode control of pneumatic systems using low-cost solenoid valves," IEEE/ASME Trans. Mechatron., vol. 12, no. 2, pp. 216-219, 2007.

[10] S. Hodgson, M. Q. Le, M. Tavakoli and M. T. Pham, "Improved tracking and switching performance of an electro-pneumatic positioning system", Mechatronics, vol. 22, no. 1, pp. 1-12, 2012.

[11] A. Grancharova and T. Johansen, "Design and comparison of explicit model predictive controllers for an electropneumatic clutch actuator using on/off valves," IEEE/ASME Trans. Mechatron., pp. 665-673, 2011.

[12] G. M. Bone, M. Xue and J. Flett, "Position control of hybrid pneumaticelectric actuators using discrete-valued model-predictive control," Mechatronics, vol. 25, pp. 1-10, 2015. 